\title{
Influencia de los reactivos y de las condiciones experimentales en la síntesis carbotérmica de nitruro de silicio
}

\author{
M.D.ALCALÁ, C.REAL, J.M.CRIADO \\ Instituto de Ciencia de Materiales de Sevilla. C.S.I.C.-UNSE. Av/Américo Vespucio s/n 41092 Sevilla, Spain
}

\begin{abstract}
Las cerámicas basadas en el $\mathrm{Si}_{3} \mathrm{~N}_{4}$ poseen unas extraordinarias propiedades físicas y mecánicas que permiten su uso como material estructural avanzado en ingeniería. Se ha utilizado la técnica de ATVC (Análisis Térmico a Velocidad de Reacción Controlada) para preparar dicho compuesto por reducción carbotérmica de sílice en atmósfera de nitrógeno y estudiar la influencia de la naturaleza de los reactivos en el producto de la reacción. Los resultados obtenidos muestran que la elección de reactivos de elevadas superficies específicas no es garantía para obtener nitruro de silicio con una morfología homogénea y de pequeño tamaño de partícula siendo recomendable el empleo de reactivos que a la temperatura de reacción posean superficies específicas lo más parecidas posibles.
\end{abstract}

Palabras claves: reducción carbotérmica, nitruro de silicio, análisis térmico.

\section{Influence of the starting materials and the experimental conditions on the carbothermal synthesis of silicon nitride}

Ceramics based on silicon nitride have unusual physical and mechanical properties that make them materials of technological importance for their use as structural material. Silicon nitride has been prepared by carbothermal reduction of silica under nitrogen atmosphere using the control rate thermal analysis (CRTA). The employ of this method allows to study the influence of the microstructure of the starting materials on the final product avoiding the influence of others experimental conditions. The results show that products of highest homogeneity and smallest particle sizes will be achieved when starting materials of similar

Key words: Carbothermal reduction, silicon nitride, thermal analysis.

\section{INTRODUCCIÓN}

A partir de la mitad del siglo XX las investigaciones sobre el nitruro de silicio cobran un inusitado auge como consecuencia del creciente interés de las cerámicas basadas en este compuesto. Este interés se debe a sus extraordinarias propiedades físicas y mecánicas que permiten su utilización en ingeniería para la fabricación de piezas de maquinarias y de herramientas de cortes de aceros duros a velocidades elevadas (1-3).

Las características químicas y físicas del polvo de nitruro de silicio son muy importantes para su posterior comportamiento durante la densificación. Los criterios más importantes empleados para determinar la calidad del polvo de nitruro de silicio son su pureza, cristalinidad, forma de las partículas, distribución del tamaño de partícula, grado de aglomeración y la razón de fases $\alpha / \beta$, así como propiedades más tecnológicas tales como la facilidad para la compactación y actividad en la sinterización (4).

Los métodos de síntesis más empleados para la obtención del polvo de nitruro de silicio son: la amonolisis de un compuesto de silicio reactivo en fase líquida o gaseosa, la reducción carbotérmica de la sílice en presencia de nitrógeno o amoniaco y la nitruración del polvo de silicio (5-19). De los métodos mencionados, la síntesis carbotérmica de $\mathrm{Si}_{3} \mathrm{~N}_{4}$ presenta la ventaja de que el material de partida es barato y de fácil disponibilidad, siendo su mayor inconveniente el contenido en impurezas procedentes de la materia prima utilizada y la elección adecuada de las condiciones experimentales (tales como materia prima, razón molar de los reactivos, composición del gas de reacción, soporte de los reactivos, diseño del reactor, temperatura y tiempos de reacción) para obtener un producto de composición y características determinadas. El mecanismo por el cual ocurre la formación del nitruro de silicio durante el proceso carbotérmico no se conoce completamente, habiéndose propuesto distintas etapas intermedias de reacción, aunque existe acuerdo en considerar al $\mathrm{SiO}$ como a un producto intermedio de la reacción (17-19).

Aunque la razón molar estequiométrica $\mathrm{C}: \mathrm{SiO}_{2}$ es $2: 1$, se requiere una razón mayor para que la síntesis sea completa. La textura de los reactivos también influye en las características del producto obtenido. Los datos, recogidos al respecto en la bibliografía, indican que el aumento de la superficie específica de los reactivos incrementa el rendimiento de la nitruración carbotérmica de la sílice (6,18-20), aún cuando exista discrepancias en cuanto a la influencia en la reactividad del tamaño inicial de partícula de la propia sílice (20). Es necesario, asimismo, una intensa homogeneización de los reactivos, en este sentido los métodos sol-gel son muy adecuados $(12,19)$, así como el uso de un reactivo natural que contenga simultáneamente carbón y sílice (16,21-22), o el empleo de polímeros como precursores (23). Debido a la reversibilidad de la reacción es preciso retirar 
el CO formado durante la síntesis, para ello se debe emplear una velocidad de flujo de nitrógeno elevada $(16,19)$. En lo que se refiere a la temperatura de reacción, se observa un considerable desacuerdo entre los diversos autores acerca de la temperatura óptima de obtención del nitruro de silicio que puede variar entre 1250 y $1550{ }^{\circ} \mathrm{C}$. Aunque la formación de $\mathrm{Si}_{3} \mathrm{~N}_{4}$ aumenta con la temperatura se debe tener en cuenta la producción de $\mathrm{SiC}$ que, dependiendo de la presión parcial de $\mathrm{CO}$, puede aparecer a partir de los $1450^{\circ} \mathrm{C}$ (9). La presión parcial de oxígeno es otro factor importante en la reducción carbotérmica de la sílice, se ha indicado la posible formación de $\mathrm{Si}_{2} \mathrm{~N}_{2} \mathrm{O}$ cuando la presión parcial de este gas es mayor que $10^{-20}$ atmósferas $(1,17,19)$.

La aplicación de la técnica de análisis térmico con velocidad de reacción controlada (ATVC) en procesos complejos, como son la síntesis carbotérmica de nitruros y carburos de silicio, ha permitido obtener productos con una composición y morfología controladas (24). Con este método, la presión parcial de $\mathrm{CO}$ se mantiene constante durante la síntesis lo que hace posible determinar la influencia de éste gas en la reacción. Por otra parte, el control de la presión parcial de $\mathrm{CO}$ conlleva el control de la velocidad a la que ocurre la reacción, eliminándose la influencia de variables experimentales tales como la cantidad de muestra empleada, la velocidad del flujo de gas utilizado, la temperatura y los tiempos de reacción. En el siguiente estudio nos proponemos analizar mediante el empleo de la técnica de ATVC la influencia de la textura y estructura de los materiales de partida y su razón molar en la síntesis carbotérmica del nitruro de silicio una vez controlados el resto de los parámetros experimentales.

\section{MATERIALES Y MÉTODOS}

\subsection{Materiales}

Los reactivos utilizados han sido dos muestras de sílice suministradas por Aldrich, 23,682-9 (S-1) y 23,678-0 (S-2), y como fuente de carbón dos muestras de carbón activo de Aldrich, 27,810-6 (C-1) y 24,227-6(C-2), y grafito suministrado por Merck, 4206(G). Los valores de superficie específica de estos materiales a temperatura ambiente y tras ser calentados durante una hora a $1400{ }^{\circ} \mathrm{C}$ en atmósfera de $\mathrm{N}_{2}$ se han incluido en la Tabla 1. Las muestras de sílice calentadas cristalizan como fase $\alpha$-cristobalita.

TABLA 1. DATOS DE SUPERFICIE ESPECÍfICA PARA LOS REACTIVOS.

\begin{tabular}{|c|c|}
\hline Muestras & $\mathbf{S}_{\text {BET }}\left(\mathbf{m}^{2} / \mathbf{g}\right)$ \\
\hline Sílice 23,682-9 (S-1) & 280 \\
Sílice 23,682-9 (S-1x) & $<1$ \\
Sílice 23,678-0 (S-2) & 430 \\
Sílice 23,678-0 (S-2x) & $<1$ \\
Carbón 27,810-6 (C-1) & 1500 \\
Carbón 27,810-6 (C-1x) & 790 \\
Carbón 24,227-6 (C-2) & 800 \\
Carbón 24,227-6 (C-2x) & 400 \\
Grafito 4206 (G) & 11 \\
Grafito 4206 (Gx) & 11 \\
\hline
\end{tabular}

$\mathrm{X}$ : indica que la muestra se calentó a $1400^{\circ} \mathrm{C}$ durante 1 hora en atmósfera de nitrógeno.

El análisis por fluorescencia de rayos $\mathrm{X}$ de las muestras de carbón mostraron un contenido de Fe del $0.42 \%$ para el grafito y de un $0,01 \%$ y $0,02 \%$ para los carbones activos C-1 y C-2, respectivamente.

El estudio termogravimétrico hasta $1400^{\circ} \mathrm{C}$ de las muestras de carbón realizados en atmósfera de $\mathrm{N}_{2}$ y haciendo pasar el gas a la salida del reactor por el detector de $\mathrm{CO}$, puso de manifiesto que los carbones sufren pérdidas de peso que se inician a temperaturas que van de $\operatorname{los} 650^{\circ} \mathrm{C}$ a los $1100^{\circ} \mathrm{C}$. De corresponder estas pérdidas únicamente a la descomposición de restos de materia orgánica adsorbida, la temperatura habría sido más baja. Cabe pensar, por tanto, que esté ocurriendo la oxidación del carbón a CO. La temperatura a la que se inicia la oxidación y la extensión en la que ésta ocurre dependen de la superficie específica de la muestra de carbón. Por esta razón, se ha considerado conveniente dar un pretratamiento a las muestras de carbón a una temperatura próxima a la de reacción $\left(1400^{\circ} \mathrm{C}\right)$ durante una hora en nitrógeno, se eliminan así los posibles restos de compuestos adsorbidos y se disminuye su superficie específica.

La oxidación del carbón es producida por el oxígeno presente en el gas portador. Este contenido depende de la permeabilidad del sistema al oxígeno de la atmósfera y varía con la velocidad del flujo de gas. Se ha podido comprobar que el uso de un $5 \%$ de $\mathrm{H}_{2}$ en el gas de reacción al tiempo que introducimos un catalizador $\left(\mathrm{Pt}-\mathrm{Al}_{2} \mathrm{O}_{3}\right)$ en el reactor logra reducir el contenido de oxígeno hasta unas $50 \mathrm{ppm}$. Por otra parte, se ha observado que la nitruración carbotérmica de sílice transcurre a menor temperatura cuando se realiza en presencia de hidrógeno, lo cual apoya la observación de Hanna y col. (15) acerca del efecto positivo del hidrógeno en la velocidad de dicha reacción. Por ello se utilizará en la síntesis una mezcla de gases compuesta por un $95 \%$ de nitrógeno y un 5\% de hidrógeno.

\subsection{Métodos}

El sistema experimental de ATVC está diseñado de tal forma que el calentamiento del horno tubular de alta temperatura (hasta $1600^{\circ} \mathrm{C}$ ) está regulado por la presión parcial de $\mathrm{CO}$ a través de una interfase (sensor de IR de CO) conectada a un controlador de temperatura de efecto PID (proporcional-integral-derivado). Para mantener constante la velocidad de reacción durante todo el proceso, el procedimiento experimental exige el control simultáneo de la presión parcial en el entorno de la muestra y del flujo gaseoso producido por la reacción. Así, si la señal captada por el detector de CO se mantiene constante en un valor prefijado por el usuario, gracias a la interfase conectada al controlador de temperatura, y la velocidad del flujo del gas reactivo y/o portador es a su vez constante, es evidente que durante la reacción la velocidad de producción del gas (velocidad de reacción) se mantiene asimismo constante. El valor de la velocidad de reacción depende, por tanto, de la señal de CO seleccionada, de la velocidad de flujo del gas y de la cantidad de muestra utilizada, variando uno de estos tres parámetros es posible elegir la velocidad de reacción obteniéndose resultados reproducibles. En la Figura 1 se muestra el registro típico de una experiencia de ATVC realizada a una presión parcial de CO de $5 \cdot 10^{-3} \mathrm{~atm}$ y una velocidad de reacción $\mathrm{C}=1,1 \cdot 10^{-3} \mathrm{~min}^{-1}$, la temperatura de corte fue $1450^{\circ} \mathrm{C}$. La representación de la fracción reaccionada, $\alpha$, frente a la temperatura se incluye, asimismo, en la Figura 1. Esta fracción se ha calculado como el cociente entre el área encerrada por la curva de $\mathrm{CO}$ a un tiempo $t$ y el área total cuando la reacción es completa.

Los productos obtenidos fueron caracterizados por DRX con un aparato PHILIPS PW 1060 provisto de contador Geiger, empleando radiación $\mathrm{Cu} \mathrm{K}_{\alpha}$ y filtro de $\mathrm{Ni}$. El tamaño del 


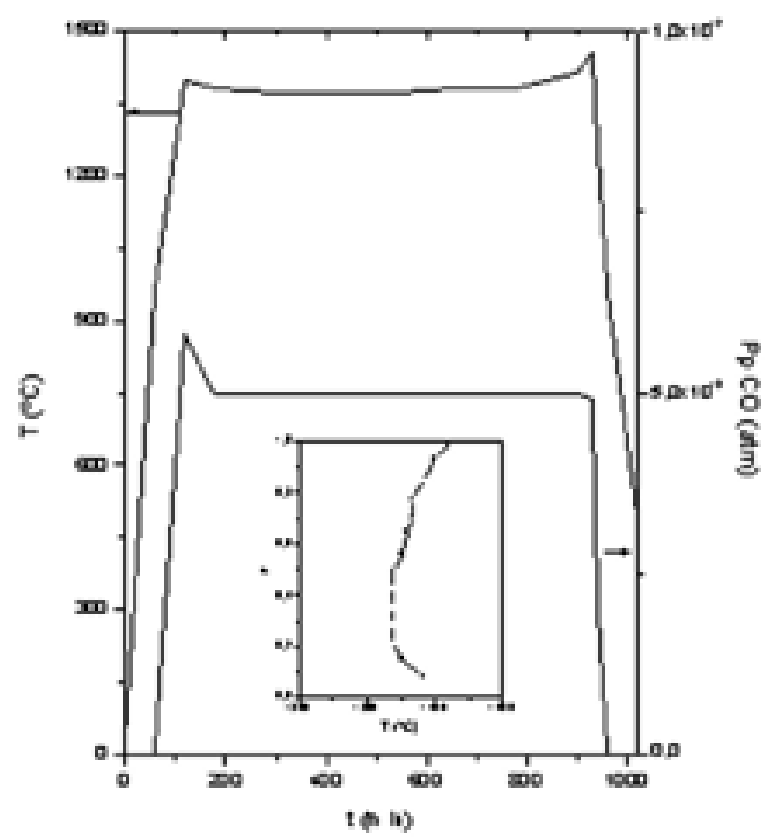

Figura 1. Gráfica correspondiente a una experiencia de ATVC realizada a una $\mathrm{P}_{\mathrm{CO}}$ de $5,0 \cdot 10^{-3}$ atmósferas y una velocidad de reacción, c, de $1,1 \cdot 10^{-3} \mathrm{~min}^{-1}$. En la gráfica insertada se muestra la curva $\alpha / T$ obtenida a partir de la gráfica anterior.

microdominio coherente de difracción de la fase $\alpha-\mathrm{Si}_{3} \mathrm{~N}_{4^{\prime}}$ que fue la mayoritaria en todos los casos, se calculó por el método de Scherrer (25) a partir de la anchura integral, corregida para la anchura instrumental, de la línea de difracción correspondiente a la familia de planos (201). El porcentaje de fases $\alpha$ y $\beta$ en el nitruro de silicio se determinó a partir de las intensidades $I_{\alpha}$ e $I_{\beta}$ correspondientes a las líneas de difracción (201) del $\alpha$ $\mathrm{Si}_{3} \mathrm{~N}_{4}$ y (101) del $\beta-\mathrm{Si}_{3} \mathrm{~N}_{4}$, respectivamente, aplicando el método de comparación directa de rayos $X$ (26). La expresión obtenida para calcular las composiciones aplicando dicho método es: $x_{\alpha}=1 /\left[1+0.668\left(I_{\beta} / I_{\alpha}\right)\right]$.

Para la determinación del área superficial $\left(\mathrm{S}_{\mathrm{BET}}\right)$ se ha utilizado el analizador rápido de superficie Micromeritics 2200.

El estudio microestructural de las muestras se realizó con un microscopio electrónico de barrido (MEB) Philips serie XL30. Las muestras se dispersaron en etanol y se depositaron sobre un soporte metálico, posteriormente se recubrieron con carbón.

Los análisis termogravimétricos se hicieron con una termobalanza Stanton Redcroft, modelo TG 770, con un límite de pesada de $100 \mathrm{mg}$ y una sensibilidad de + / - 0.5 microgramos.

\section{RESULTADOS Y DISCUSIÓN}

El uso de la técnica de ATVC para preparar nitruro de silicio por reducción carbotérmica de la sílice en atmósfera de $\mathrm{N}_{2}$ reduce considerablemente el número de variables experimentales que deben ser ajustadas para la optimización del proceso, quedando estas reducidas prácticamente a la textura, estructura y razón molar de los reactivos sólidos.

Los trabajos recogidos en la bibliografía muestran preferencia por el uso de naves de grafito para realizar la síntesis del nitruro de silicio. La razón es que el otro material que se suele emplear, la alúmina, podría favorecer (19) la formación de oxini- truro como subproducto de reacción, debido a un aumento en la presión parcial de oxígeno en el entorno de la muestra $\left(\mathrm{Al}_{2} \mathrm{O}_{3(\mathrm{~s})}\right.$ ---> $\mathrm{Al}_{2} \mathrm{O}_{2(\mathrm{~g})}+1 / 2 \mathrm{O}_{2(\mathrm{~g})}$ ). Sin embargo, la presión parcial de $\mathrm{O}_{2}$ producida por esta reacción, $10^{-22}$ atmósferas, es despreciable frente a la presión parcial de oxígeno existente en el interior del reactor. Por otro lado, las naves de grafito muestran un deterioro progresivo tras su uso que sugiere su oxidación a lo largo de la reacción. En estas condiciones parte del $\mathrm{CO}$ detectado en la experiencia de ATVC procede de dicha nave, lo que dificulta el control de la velocidad de reacción, los tiempos de reacción son mayores y las velocidades más lentas de lo previsto. Como consecuencia, las experiencias realizadas en nave de grafito son difíciles de reproducir y por todo ello se ha preferido utilizar naves de alúmina para realizar este estudio.

La mezcla de los reactivos se realizó con un mortero de ágata y siempre se utilizaron unos $0.9 \mathrm{~g}$ de sílice, pudiendo variar el contenido en carbón. La velocidad de flujo del gas de reacción (mezcla de un 95\% de nitrógeno y un 5\% de hidrógeno) fue de $200 \mathrm{cc} / \mathrm{min}$ y se trabajó con una presión parcial de $\mathrm{CO}$ de $5 \cdot 10^{-3} \mathrm{~atm}$. Estas condiciones experimentales determinaron una velocidad de reacción de $1.1 \cdot 10^{-3} \mathrm{~min}^{-1}$. La temperatura máxima de corte, a fin de evitar la formación de SiC, se fijó en $1450^{\circ} \mathrm{C}$.

Como se ha indicado en la introducción, para que la conversión de $\mathrm{SiO}_{2}$ a $\mathrm{Si}_{3} \mathrm{~N}_{4}$ de acuerdo con la reacción (1) sea completa,

$$
3 \mathrm{SiO}_{2}+6 \mathrm{C}+2 \mathrm{~N}_{2} \rightarrow \mathrm{Si}_{3} \mathrm{~N}_{4}+6 \mathrm{CO}
$$

es necesario usar una relación molar $\mathrm{C}: \mathrm{SiO}_{2}$ mayor que la teórica, 2. Este hecho se ha justificado en la bibliografía por la necesidad de un exceso de carbón para reducir la presión parcial de $\mathrm{O}_{2}$ durante la reacción evitando la formación de oxinitruro $(17,27)$, o para favorecer la formación y reducción del intermedio de reacción $\mathrm{SiO}(6,18,20)$. Algunos autores consideran que el aumento de la velocidad de reducción carbotérmica de la sílice observado al aumentar la razón molar $\mathrm{C}: \mathrm{SiO}_{2}$ se debe al aumento implícito de la cantidad total de hierro, habitualmente presente como impureza en el carbón (5). En el presente estudio para determinar este parámetro, se realizaron tres experiencias (I-III) con razones molares de reactivos diferentes (Tabla 2). El contenido en hierro del C-1 es de un $0.01 \%$ en peso lo cual elimina el posible efecto catalítico del citado metal.

TABLA 2. RELACIÓN DE INTENSIDADES DE DIFRACCIÓN $I_{\beta} / I_{\alpha}$, PARA LOS PICOS DE DIFRACCIÓN (201) DE LA FASE $\alpha$ Y (101) DE LA FASE $\beta$, Y DATOS DEL PORCENTAJE DE FASE A FORMADA, DEL TAMAÑO COHERENTE DE DIFRACCIÓN PARA DICHA FASE Y DE LA SUPERFICIE ESPECÍFICA DE LOS PRODUCTOS OBTENIDOS.

\begin{tabular}{|c|c|c|c|c|c|c|}
\hline Experiencia & Reactivos & $\mathrm{C} / \mathrm{SiO}_{2}$ & $\mathbf{I}_{\beta} / \mathbf{I}_{\alpha}$ & $\% \alpha-\mathbf{S i}_{3} \mathbf{N}_{4}$ & $\mathrm{D}(\mathrm{nm})$ & $\mathrm{S}_{\mathrm{BET}}\left(\mathrm{m}^{2} / \mathrm{g}\right)$ \\
\hline I & S-2+C-1x & 2.7 & --- & $36(\alpha), 64\left(\mathrm{SiO}_{2}\right)$ & --- & --- \\
\hline II & $S-2+C-1 x$ & 3.4 & 0.045 & 97 & 84 & $<1$ \\
\hline III & $S-2+C-1 x$ & 5.5 & 0.062 & 96 & 32 & 1.4 \\
\hline IV & $S-2 x+C-1 x$ & 5.5 & 0.062 & 97 & 43 & $<1$ \\
\hline V & $S-1+C-1 x$ & 5.5 & 0.062 & 97 & 53 & $<1$ \\
\hline VI & $S-1 x+C-1 x$ & 5.5 & 0.070 & $\begin{array}{l}96 \\
92\end{array}$ & 47 & 5.1 \\
\hline VII & $S-1 x+C-2 x$ & 5.5 & 0.150 & $\begin{array}{c}92 \\
49(\alpha) 16(\beta)\end{array}$ & 73 & 4.6 \\
\hline VIII & $S-1 x+G x$ & 5.5 & 0.510 & $\begin{array}{c}49(\alpha), 16(\beta) \\
35(\mathrm{SiC})\end{array}$ & 43 & 8.1 \\
\hline
\end{tabular}

Las curvas $\alpha-T$ correspondientes a estas experiencias se han incluido en la Figura 2a. Puede observarse que el aumento de la razón molar $\mathrm{C}: \mathrm{SiO}_{2}$ produce un descenso de la temperatura a la cual transcurre la reacción. De la caracterización de los productos por DRX se obtuvieron los resultados de composición y de tamaños de microdominio coherente de 
difracción que se muestran en la Tabla 2. En esta tabla también se han incluido los datos de superficie específica de los productos. Puede observarse en dicha tabla, que al aumentar la razón molar $\mathrm{C}: \mathrm{SiO}_{2}$ hasta 3.4, aumenta la conversión de $\mathrm{SiO}_{2}$ en $\mathrm{Si}_{3} \mathrm{~N}_{4}$. A partir de ese valor la reacción es completa y no se modifica el porcentaje de fases $\alpha$ y $\beta$ presentes en el producto de reacción aunque sí se obtienen menores tamaños de microdominio coherente de difracción y una mayor superficie específica, en acuerdo con las consideraciones realizadas en la bibliografía (5-6,19-20). La morfología del nitruro de silicio obtenido de las experiencias II y III (Figura 2b), donde la conversión fue completa, está constituida por clusters de cristales de apariencia de prisma hexagonal apreciándose una menor distribución de tamaños de los mismos cuando se trabaja con un mayor contenido de carbón.

Como conclusión de los resultados anteriores se decidió emplear, en el estudio de la influencia de la textura y estructura de los reactivos (Tabla 1) en la síntesis carbotérmica del nitruro de silicio, un valor para la razón molar C: $\mathrm{SiO}_{2}$ de 5.5. En primer lugar, se consideró la influencia de la sílice en el producto de la reacción. Los resultados obtenidos corresponden a las experiencias III-VI (Tabla 2). Las curvas $\alpha / T$ representadas en la gráfica de la Figura 3a muestran que cuando la reacción se lleva a cabo con las muestras de sílice cristalizadas (S-1x y S-2x) transcurre a menor temperatura. Los datos de composición de las fases $\alpha$ y $\beta$ del $\mathrm{Si}_{3} \mathrm{~N}_{4}$ y los tamaños de microdominio coherente de difracción para la fase mayoritaria, $\alpha$, obtenidos a partir de la caracterización por DRX de los productos, junto a las superficies específicas de los mismos se han incluido en la Tabla 2. Puede observarse como estas propiedades de los productos no se ven prácticamente afectadas por las características texturales y estructurales de la sílice utilizada, al menos en las condiciones empleadas en este estudio para realizar la mezcla de los reactivos. Únicamente el producto obtenido a partir de la sílice S-1x (VI) presenta una mayor superficie específica. En la Figura $3 b$ se muestra su microfotografía MEB, donde podemos comprobar que posee una morfología similar a la del producto obtenido a partir de la muestra de sílice S-2 (Figura 2b, III).

Los resultados obtenidos parecen indicar que la superficie específica inicial de la sílice y su estructura no influyen prácticamente en el tamaño cristalino del producto de la reducción carbotérmica, alcanzándose el mismo resultado si se parte de una sílice amorfa o cristalina. La razón puede estar en el hecho de que cuando se inicia la reacción la sílice se encuentra ya cristalizada, no siendo suficiente la presencia del carbón durante el calentamiento para evitar la sinterización de las partículas de sílice. El recubrimiento de las partículas sinterizadas por carbón queda garantizado al conservar éste gran parte de su superficie específica a dicha temperatura (el C-1 a la temperatura de reacción tiene una superficie específica de unos $800 \mathrm{~m}^{2} / \mathrm{g}$ ).

Atendiendo a los resultados recogidos en el apartado anterior se decidió utilizar la muestra de sílice S-1x para estudiar la influencia de la textura y estructura del carbón en la reacción carbotérmica. Las experiencias correspondientes a este estudio son las VI-VIII de la Tabla 2. Las representaciones $\alpha / T$ incluidas en la Figura 4a muestran que no hay un cambio significativo en la temperatura de reacción cuando se usan carbones activos con distintas superficies específicas. Este hecho no está de acuerdo con los datos reflejados en la bibliografía que indican un aumento de la velocidad de reacción con la superficie específica del carbón (18-20). Si se tiene en cuenta que la superficie específica de la sílice a la temperatura de reacción es $<1 \mathrm{~m}^{2} / \mathrm{g}$ (Tabla 1), lo que puede ocurrir es que al aumentar la superficie específica del carbón lejos de favorecerse el contacto entre las partículas de $\mathrm{C}$ y de $\mathrm{SiO}_{2}$, éste disminuya. En este sentido Ekelund y Forslund (6) han indicado que no es suficiente con aumentar la superficie específica de uno de los reactivos para a)

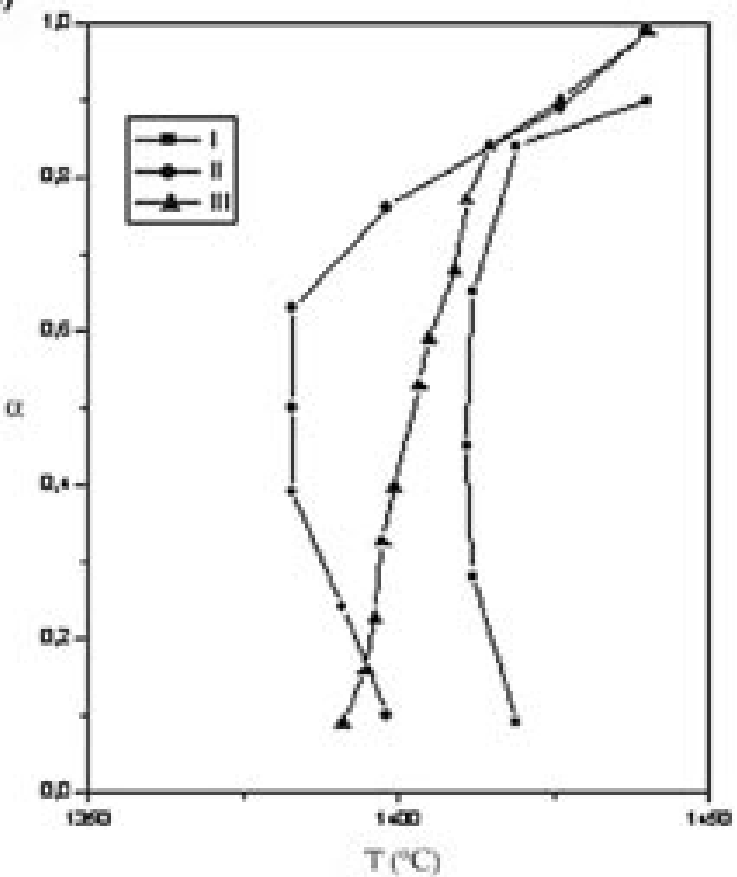

b)

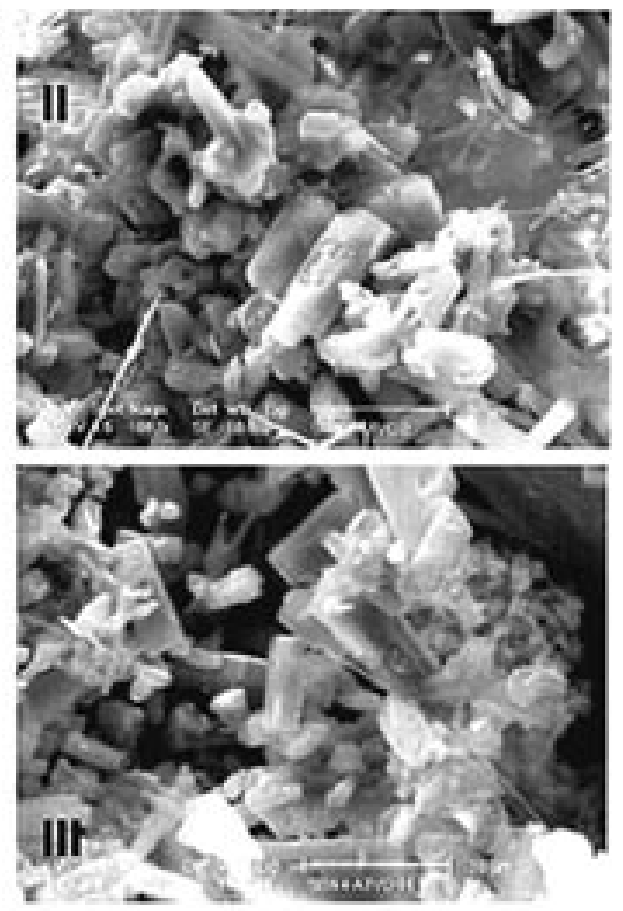

Figura 2. a) Curvas $\alpha / T$ obtenidas para las experiencias de ATVC I-III de la Tabla 2; b) microfotografías MEB de las muestras procedentes de las experiencias II y III. 
mejorar las condiciones de reacción, debe también tenerse en cuenta la superficie del otro reactivo implicado. De hecho, en la misma figura se puede observar que cuando se utiliza grafito la reacción transcurre a una temperatura inferior. En este último caso, la diferencia entre las superficies específicas de los reactivos es menor y, además, por tratarse el grafito de un material blando cabe esperar que el grado de intimidad alcanzado en la mezcla mecánica con la sílice sea mayor que con los carbones activos. En este sentido se debe indicar que tanto las reducciones carbotérmicas de mezclas de sílice y carbón procedentes de cascarillas de arroz purificadas (22) como de mezclas artificiales homogeneizadas en molinos de bolas (28), donde el grado de intimidad entre los reactivos es mayor, transcurren a temperaturas más bajas.

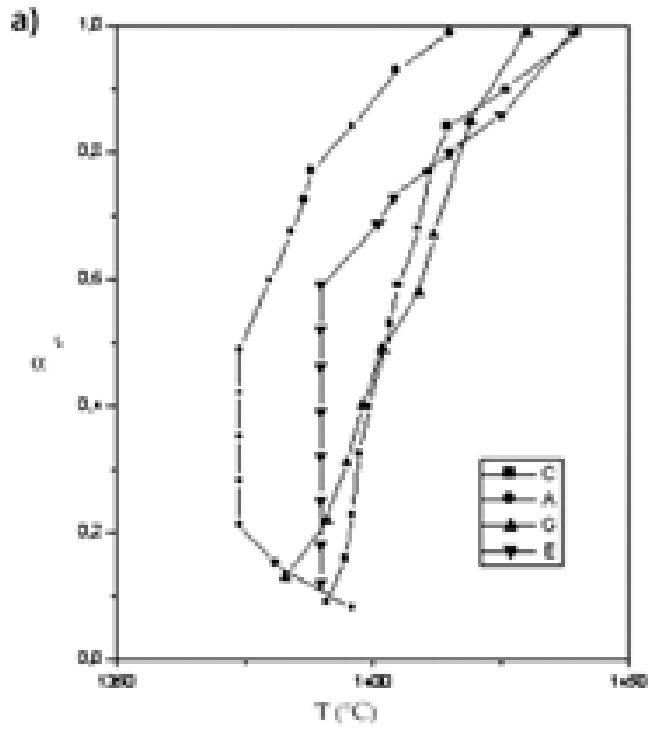

Figura 3. a) Curvas $\alpha /$ T obtenidas para las experiencias de ATVC III-VI de la Tabla 2; b) microfotografía MEB de la muestra procedente de la experiencia VI.

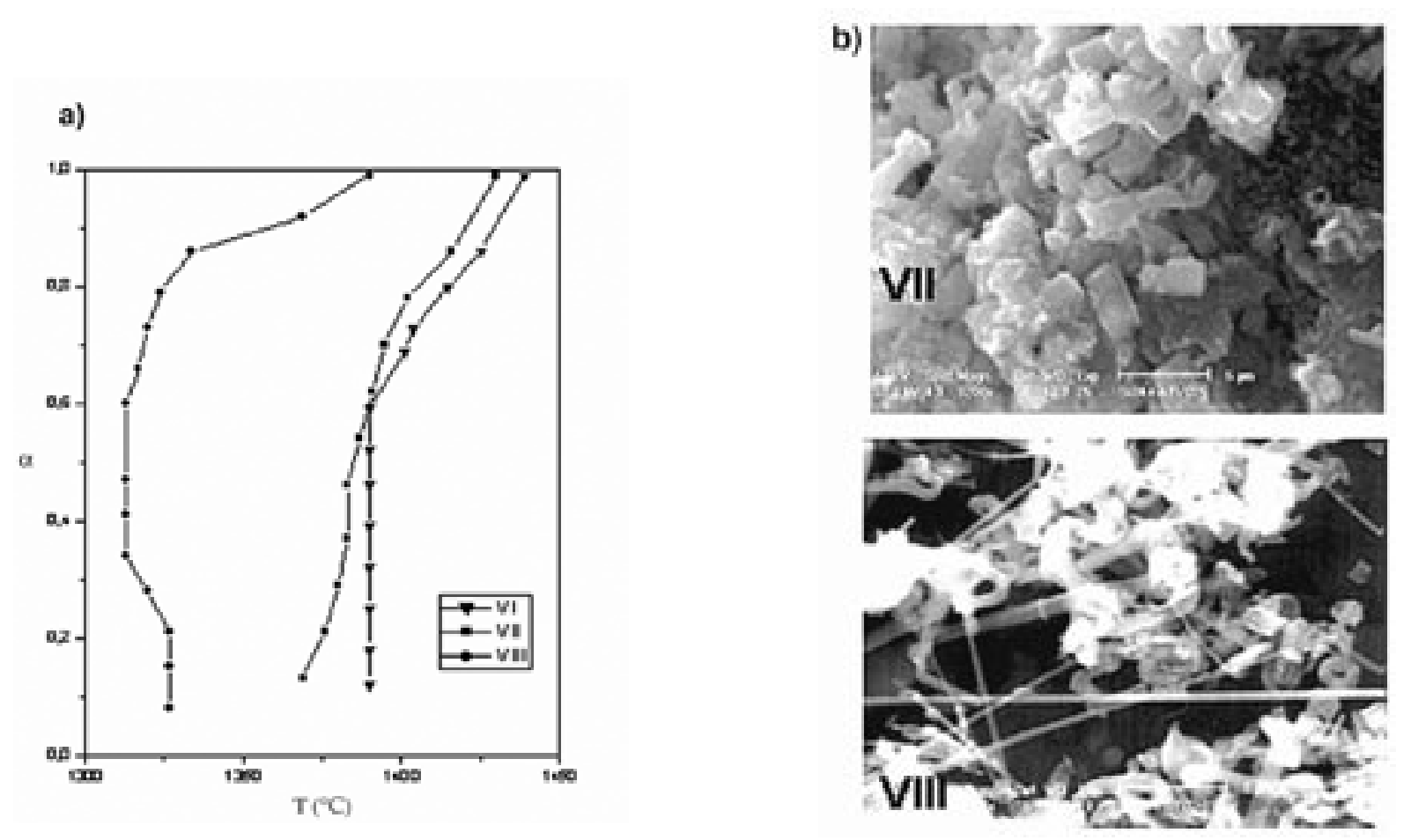

A partir de los datos correspondientes de la DRX y del mismo modo que se hizo con la serie anterior, se determinó la composición del producto sintetizado y el tamaño de microdominio coherente de difracción para la fase mayoritaria, $\alpha$ $\mathrm{Si}_{3} \mathrm{~N}_{4}$. Los resultados obtenidos se han incluido en la Tabla 2, junto a los valores de la superficie específica de cada producto. En el caso de la muestra preparada a partir de grafito (VIII), se ha observado la presencia en el producto de reacción de carburo de silicio además de una mayor cantidad de fase $\beta-\mathrm{Si}_{3} \mathrm{~N}_{4}$. La formación de ambos compuestos puede estar determinada por el contenido de $\mathrm{Fe}(0.42 \%)$ en esta muestra de carbón $(6,17,27)$. El efecto catalítico $(6,17)$ de esta impureza puede ser, asimismo, otra de las causas del descenso tan acusado de la temperatura de reacción observada en este caso.

En la Figura 4 b se incluyen las microfotografías MEB de los productos de las experiencias VII y VIII. El producto obtenido a partir del carbón de menor superficie específica (C-2x), está formado por cristales con forma de prismas hexagonales más

b)

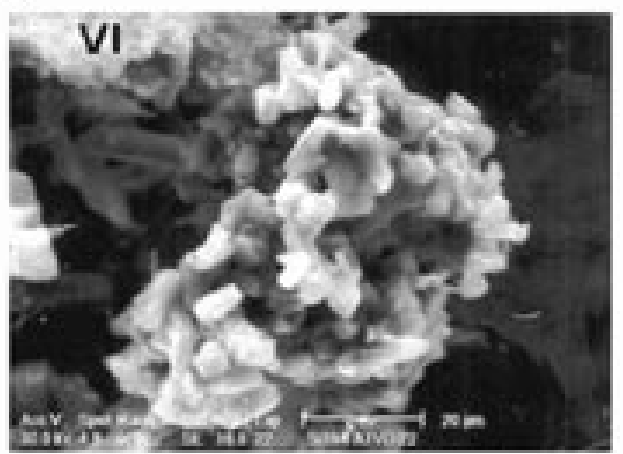

Figura 4. a) Curvas $\alpha / T$ obtenidas para las experiencias de ATVC VI-VIII de la Tabla 2; b) microfotografías MEB de las muestras procedentes de las experiencias VII y VIII. 
regulares y pequeños que los correspondientes del obtenido empleando el C-1x (Figura 3b). Este hecho puede estar motivado, como ya se ha indicado, por un mejor contacto de los reactivos cuando se usa el C-2x ya que a la temperatura de reacción este posee la mitad de superficie específica que el C-1x, lo que además determinó que la experiencia VII transcurriera a menor temperatura.

La morfología del producto obtenido a partir del grafito es completamente distinta a las anteriores, observándose la formación de whiskers y pequeños cristalitos. La ausencia de esferas en los extremos de las fibras, que han sido observadas en la obtención de $\mathrm{SiC}$ empleando los mismos reactivos que en el presente estudio (29), sugiere un mecanismo de reacción, al menos al inicio, vapor-sólido (VS) en el que no está presente una fase líquida y que ha sido propuesto para la formación de $\alpha-\mathrm{Si}_{3} \mathrm{~N}_{4}(22,30)$. La aparición de fibras de $\alpha-\mathrm{Si}_{3} \mathrm{~N}_{4}$ ha sido observada en las superficies libres de la mezcla de reactivos relacionándose con el empleo de carbones de pequeña superficie específica, que permiten la obtención de mezclas porosas de $\mathrm{C}$ y SiO $(5,9)$. En el presente estudio, la estructura laminar del grafito ha podido favorecer dicha formación.

Es interesante resaltar que en ninguna de las experiencias realizadas se ha observado la formación de la fase $\mathrm{Si}_{2} \mathrm{~N}_{2} \mathrm{O}$ a pesar de que el contenido en oxígeno en el sistema durante la reacción fuera, como ya se ha indicado, mayor al valor de $10^{-20}$ atmósferas requerido para la estabilización de la citada fase $(1,17,19)$. A este respecto, Siddiqui y Hendry (17) han indicado la necesidad de una fase líquida para que se forme éste compuesto.

\section{CONCLUSIONES}

Los resultados obtenidos en el presente trabajo muestran que la elección de reactivos de elevadas superficies específicas no es garantía suficiente para obtener polvo de nitruro de silicio con una morfología homogénea y de pequeño tamaño de partícula a partir de la reducción carbotérmica de sílice en atmósfera de nitrógeno. Dado que las partículas de sílice han sinterizado cuando se inicia la reacción, es indiferente la superficie específica de partida que posea este reactivo, alcanzándose el mismo resultado si se parte de una sílice amorfa o cristalina. Quizás podría profundizarse en este aspecto empleando una técnica de homogeneización para la mezcla de los reactivos más adecuada que la empleada en este caso, como podría ser el uso de un molino de bolas y que en un estudio anterior se ha mostrado eficaz para descender la temperatura de la nitruración carbotérmica de la sílice, influyendo además en la morfología del producto final (28). En lo que se refiere al carbón es recomendable el uso de una muestra que a la temperatura de reacción posea una superficie específica lo más próxima posible a la de la sílice. El uso de grafito se desaconseja ya que su estructura laminar favorece la obtención de un producto con una morfología heterogénea. La pureza de los reactivos debe ser rigurosamente controlada si no se quieren obtener mezclas de compuestos en el producto de la reacción.

El contenido de oxígeno en el reactor no depende de la pureza del gas empleado o del tipo de soporte que se utilice para los reactivos sino del diseño del dispositivo. El uso de un porcentaje pequeño de $\mathrm{H}_{2}$ y la introducción de un catalizador en el reactor ayudan a su control. Probablemente se encuentre en este hecho la necesidad de utilizar un exceso de carbón que ayude a mantener baja la presión parcial de oxígeno en el entorno de la muestra y que dependerá, en cada caso, del dispositivo empleado para llevar a cabo el proceso.

La elección adecuada de los reactivos y el uso de la técnica de ATVC para controlar la morfología y composición del producto de reacción, pueden eliminar los inconvenientes que presenta la nitruración carbotérmica para obtener a partir de reactivos de fácil disponibilidad cerámicas de altas prestaciones basadas en el nitruro de silicio.

\section{BIBLIOGRAFÍA}

1. M. Taguchi. "Applications of high technology ceramics in Japonese Automobiles". Adv. Ceram. Mater. 2 [4] 754-62 (1987).

2. H. Gleiter. "Nanocrystalline materials". Prog. Mater. Science, 33 223-315 (1989).

3. R. Barea, P. Miranzo, M. I. Osendi. "Silicon nitride - superalloyis joining". Bol. Soc. Esp. Ceram. Vidr. 39 [5] 647-51 (2000).

4. W. H. Rhodes and S. Natausohn. "Powders for advanced structural ceramics". Ceram. Bulletin 68 [10] 1804-12 (1989).

5. F. R. Van Dijen and U. Vogt. "The chemistry of the carbothermal synthesis of $\alpha-\mathrm{Si}_{3} \mathrm{~N}$ Reaction mechanism, reaction rate and properties of the product". J. Eur. Ceram. Soc. 10 273-82 (1992).

6. M. Ekelund and B. Forslund. "Carbothermal preparation of silicon nitride: Influence of starting materias and synthesis parameters". J. Am. Ceram. Soc. 75 [3] 532-39 (1992).

7. M. Barsoum, P. Kangutkar and M.J. Koczak. "Nitridation kinetics and thermodynamics of silicon powder compacts". J. Am. Ceram. Soc. 74 [6] 1248-53 (1991).

8. I. G. Cano and M. A. Rodríguez. "Synthesis of $\mathrm{Si}_{3} \mathrm{~N}_{4}$ ”. Bol. Soc. Esp. Ceram. V. 42 [2] 89-92 (2003).

9. H. Lange, G. Wötting and G. Winter. "Silicon nitride - from powder synthesis to ceramic materials". Angew. Chem. Int. Ed. Engl. 30 1579-97 (1991).

10. A. J. Moulson. "Review. Reaction-bonded silicon nitride: Its formation an properties". J. Mater. Sci. 14 1017-51 (1979).

11. G. Ziegler, J. Heinrich and G. Wötting. "Review. Relationships between processing, microstructure and properties of dense and reaction-bonded silicon nitride". J. Mater. Sci. 22 3041-86 (1987).

12. D. L. Segal. "A review of preparative routes to silicon nitride powders". Br. Ceram. Trans. J. 85 184-87 (1986).

13. T. Hashishin, Y. Kaneko, H. Iwanaga and Y. Yamamoto. "The synthesis of silicon nitride whiskers from $\mathrm{SiO}_{2}-\mathrm{N}_{2}-\mathrm{Na}_{3} \mathrm{AlF}$ system". J. Mater. Sci. 34 2193-97 (1999).

14. A. W. Weimer, G. A. Eisman, D. W. Susnitzky, D. R. Beaman and J. W. MaCoy. "Mechanism and kinetics of the carbothermal nitridation synthesis of $\alpha$-silicon nitride". J. Am. Ceram. Soc. 80 [11] 2853-63 (1997).

15. S. B. Hanna, N. A. L. Mansour, A. S. Taha and H. M. A. Abd-Allah. "Silicon carbide and nitride from rice hulls. III-Formation of silicon nitride". Br. Ceram. Trans. J. 84 18-21 (1985).

16. A. Rahman and F. L. Riley. "The control of morphology in silicon nitride powder prepared from rices husk". J. Eur. Cer. Soc. 5 11-22 (1989).

17. S.A. Siddiqi and A. Hendry. "The influence of iron on the preparation of silicon nitride from silica". J. Mater. Sci. 20 3230-38 (1985).

18. M. Komeya and H. Inove. "Synthesis of the $\alpha$-form of silicon nitride from silica". J. Mater Sci. Lett. 10 1243-46 (1975).

19. S. J. P. Durham, K. Shanker and R. A. L. Drew. "Carbothermal synthesis of silicon nitride Effect of reaction conditions". J. Am. Ceram. Soc. 74 [1] 31-37 (1991).

20. Shi-Chang Zhang and W. Roger Cannon. "Preparation of silicon nitride from silica". J. Am. Ceram. Soc. 67 [10] 691-95 (1984).

21. Y. Mizuhara, M. Nogochi, T. Ishihara, A. Saton, K. hiranatsu and Y. Takita. "Preparation of fiberlike silicon-nitride from diatomaceous-earth". J. Am. Ceram. Soc. 74 [4] 846-48 (1991).

22. C. Real, M. D. Alcalá and J. M. Criado. "Synthesis of silicon nitride from carbothermal reduction of rice husks by the CRTA method". J. Am. Ceram. Soc. 87 [1] 75-78 (2004).

23. D. R. Stanley, J. D. Birchall, J. N. Kenneth Hyland, L. Thomas and K. Hodgetts. "Carbothermal synthesis of binary $(\mathrm{MX})$ and ternary $\left(\mathrm{M}^{1}, \mathrm{M}^{2} \mathrm{X}\right)$ carbides, nitrides and borides from polymeric precursors". J. Mater. Chem. 2 [2] 149-56 (1992).

24. M. D. Alcalá, J. M. Criado and C. Real. "Sample Controlled Reaction Temperature (SCRT): Controlling the phase composition of silicon nitride obtained by carbothermal reduction". Adv. Eng. Mater. 4 [7] 478-82 (2002).

25. L. E. Alexander, H. P. Klung. "X-Ray Diffraction Procedures", pp. 618 Ed. John Wiley \& Sons, New York 1974.

26. B. D. Cullity. “Elements of X-Ray Diffraction", pp. 397 Ed. Assison-Wesley Publ. Co., London 1978.

27. F. F. Lange. "Polyphase systems: Fabrication, microstructure and properties". International Metals Review, 11-20 (1980).

28. M.D. Alcalá, J.M. Criado and C. Real. "Influence of the experimental conditions and the grinding of the starting materials on the structure of silicon nitride synthesis by carbothermal reduction". Solid State Ionics 141-142 1657-1661 (2001).

29. C. Real, M. D. Alcalá and J. M. Criado. "Synthesis of silicon carbide whiskers from carbothermal reduction of silica gel by means of the constant rate thermal analysis (CRTA) method". Solid State Ionics 95 29-32 (1997).

30. S. Shimada and T. Kataoka. "Separate growth of $\alpha$ and $\beta \mathrm{Si}_{3} \mathrm{~N}_{4}$ whiskers on or near a carbon substrate by carbothermal reduction". J. Am. Ceram. Soc. 84 [10] 2442-44 (2001).

Recibido:

Aceptado: 